\title{
1 Community Cycling Exercise for Stroke Survivors is Feasible and \\ 2 Acceptable
}

3 Authors: Andy Kerr $\mathrm{PhD}^{\mathrm{a}^{*}}$, Joanne Cummings $\mathrm{PhD}^{\mathrm{b}}$, Mark Barber $\mathrm{MD}^{\mathrm{c}}$,

4 Marie McKeown $\mathrm{BSc}^{\mathrm{d}}$, Phillip Rowe $\mathrm{PhD}^{\mathrm{a}}$, Gillian Mead MD ${ }^{\mathrm{e}}$, Amy

5 Doucet $\mathrm{MSc}^{\mathrm{b}}$, Katherine Berlouis $\mathrm{MSc}^{\mathrm{b}}$, Madeleine Grealy $\mathrm{PhD}^{\mathrm{b}}$

$6 \quad{ }^{a}$ Department of Biomedical Engineering, University of Strathclyde, Glasgow, United

7 Kingdom

$8{ }^{b}$ School of Psychological Sciences and Health, University of Strathclyde, Glasgow,

9 United Kingdom

$10{ }^{c}$ Department of Medicine for the Elderly, NHS Lanarkshire, Airdrie, United Kingdom

11 South Lanarkshire Leisure and Culture, Hamilton, United Kingdom

$12{ }^{e}$ Centre for Clinical Brain Sciences, University of Edinburgh, Edinburgh, United

13 Kingdom

14 Corresponding author

15 Dr Andy Kerr, Department of Biomedical Engineering, University of Strathclyde, G1

16 1QE, United Kingdom.

17 Tel: +44 (0)141 548 2855, Email: a.kerr@strath.ac.uk

18 Orchid: 0000-0002-7666-9283

19 The trial was registered: UKCRN - ID 17583.

20 Acknowledgement

21 The authors would like to acknowledge funding from the Chief Scientist

22 Office $(\mathrm{CZH} / 4 / 1006)$ to carry out aspects of this study. We would also like

23 to express our sincere thanks to the management and staff of Lanarkshire 
24 Sport and Leisure services for their support and enthusiasm for the study 25 without which it could not have been conducted. 


\section{Community Cycling Exercise for Stroke Survivors is Feasible and \\ 27 Acceptable}

28 Abstract

29 Background

30 Physical activity is recommended after stroke but levels for stroke survivors are

31 typically low. The use of indoor recumbent cycling, delivered through local government

32 leisure facilities, may increase access to exercise among stroke survivors.

33 Objective

34 This study aimed to evaluate the acceptability and feasibility of an indoor cycling

35 programme delivered through existing local government services.

36 Methods

37 Participants were recruited through stroke liaison nurses and public advertising. After a

38 home visit to assess eligibility and conduct psychological and general health

39 assessments, participants attended their local leisure centre for an initial fitness test and

40 short battery of physical tests. Then an eight week training programme was designed

41 with weekly goals. Following the programme the assessments were retaken along with

42 an evaluation questionnaire. In-depth, semi-structured, interviews were conducted with

$43 \quad 15$ participants and 5 fitness coaches.

44 Results

45115 individuals volunteered to participate during a 10 month recruitment period, 77 met

46 the inclusion criteria and consented, 66/77 (86\%) completed the programme including

47 all eight non-ambulatory participants. The programme and procedures (recruitment and

48 outcome measures) were feasible and acceptable to participants (81\% reported

49 following the programme). Participants were generally very positive about the

50 experience. Significant improvements in sit-to-stand capacity $\left(\mathrm{M}_{\text {pre }}=25.2 \mathrm{~s}, \mathrm{M}_{\text {post }}=19.0 \mathrm{~s}\right.$,

$51 \mathrm{p}=.002)$, activities of daily living (NEADL, $\left.\mathrm{M}_{\mathrm{pre}}=12.2, \mathrm{M}_{\mathrm{post}}=13.2, \mathrm{p}=.002\right)$,

52 psychosocial functioning (SAQOL, $\mathrm{M}_{\text {pre }}=3.82, \mathrm{M}_{\text {post }}=4.15, \mathrm{p}=.001$ ), energy (SAQOL, 
$\left.53 \mathrm{M}_{\mathrm{pre}}=3.75, \mathrm{M}_{\mathrm{post}}=4.02, \mathrm{p}=.018\right)$ and depression (GHQ, $\left.\mathrm{M}_{\mathrm{pre}}=.97, \mathrm{M}_{\mathrm{post}}=.55, \mathrm{p}=.009\right)$

54 were observed.

55 Conclusion

56 A cycling based exercise programme delivered through local leisure centre staff and

57 facilities was shown to be feasible and acceptable for people living with stroke.

58

59 Keywords (3 to 5): Stroke, Exercise, Quality of Life, Physical Fitness

60

61 Abbreviations

62 MRC: Medical Research Council

63 STS: Sit to stand

64 FTSTST: Five Times Sit-to-Stand Test

65 PWS: Preferred walking speed

66 6MWT: Six-Minute Walk Test

67 NEADL: Nottingham Extended Activities of Daily Living Scale

68 SAQOL-39: The Stroke and Aphasia Quality of Life Scale

69 GHQ-28: The General Health Questionnaire

70 PSQI: The Pittsburgh Sleep Quality Index

71 MoCA: Montreal Cognitive Assessment

72 FAS: The Fatigue Assessment Scale

73 FITT: Frequency, Intensity, Type and Time 


\section{Introduction}

79 Physical activity after stroke improves fitness and capacity to carry out daily activities, with growing evidence for positive cognitive ${ }^{1,2}$ and quality of life outcomes. ${ }^{3}$ This evidence has been produced consistently across a wide range of high quality studies ${ }^{4}$ using well controlled methodologies. The same body of literature, however, reports the persistence of very low levels of physical activity among stroke survivors ${ }^{5,6}$ suggesting difficulties translating research evidence into the real world.

Whilst adherence to physical activity programmes among stroke survivors participating in research trials can be high, for example Mead et al. ${ }^{7}$ reported $100 \%$ attendance for $59 \%$ of participants, this positive response may not reflect experiences outside the controlled environment of a research trial where high levels of resource and support are common. Taking advantage of local government funded leisure facilities may be a more acceptable and economically sustainable model, albeit with the continued involvement of health professionals which has been shown to improve attendance amongst individuals with poorer physical function. ${ }^{8}$

93 Indoor cycling, particularly with semi-recumbent cycle ergometers, offers specific

94 advantages for stroke survivors attempting to exercise. These ergometers provide a safe stable environment with adaptable positions for wheelchair users and an ability to make incremental changes to cadence and resistance to tailor exercise. The use of cycle ergometers with stroke survivors has been reported to be safe and acceptable with evidence of improvements to balance, strength, general function and motor control. ${ }^{9,} 10$

99 These findings can, at least in part, be explained by the similarity (kinematics and muscle activation) between recumbent cycling and the functional tasks of climbing a

101 step and rising from a chair. ${ }^{11}$ 
This study investigated the feasibility of community dwelling stroke survivors participating in a cycling based exercise programme delivered through existing local

104 government funded staff and facilities. The primary aim was to assess feasibility and acceptability, this comprised recruitment, assessments, adherence and acceptability of

106 the exercise programme from both the participants and fitness coaches' perspective.

107 Secondly, to collect evidence of any change in outcome measures to inform future 108 controlled trials.

\section{Material and Methods}

110 This was a mixed methods feasibility trial with a 'pre-post' intervention quasi-

111 experimental design (UKCRN - ID 17583) based on Medical Research Council (MRC)

112 guidelines for the development of complex interventions. ${ }^{12}$ The methods conform to the

113 STROBE Guidelines (see supplementary information).

\section{Participants}

115 Participants were medically stable stroke survivors resident within Lanarkshire

116 (Scotland) local council area. The local councils are responsible for providing local

117 services and facilities, including sport and leisure.

118 Participants responded to verbal invitations from a stroke liaison nurse who

119 routinely see patients during the 12 month period following discharge and recruitment

120 posters placed in community locations and local newspapers. The study was approved

121 by the East of Scotland Research Ethics Committee (14/ES/1080). Individuals interested

122 in participating were invited to contact the research group who screened for eligibility,

123 using the following criteria, and then sent an information package. The recruitment

124 period lasted 10 months (01/12/2014 until 30/09/2015).

125 Inclusion criteria; a diagnosis of stroke (physician confirmed), over 18 years old

126 and resident in Lanarkshire. Exclusion criteria included; contraindications to physical 
127 activity, a recent injurious fall without medical assessment, cognitive impairment severe

128 enough to prevent protocol adherence, assessed with the Montreal Cognitive

129 Assessment (MoCA $)^{13}$ and advice from the clinical team.

\section{$130 \quad$ Materials}

\section{$131 \quad$ Physical capacity}

132 Four tests of physical capacity were conducted: 1) Five Times Sit-to-Stand Test

133 (FTSTST) ${ }^{14}$ where the time to perform 5 sit to stand movements is recorded, 2)

134 preferred walking speed (PWS), timed over 5 meters $^{15}$, 3) Six-Minute Walk Test

135 (6MWT) using a standard treadmill ${ }^{16}$, where the distance walked during 6 minutes is

136 recorded and 4) the Nottingham Extended Activities of Daily Living Scale (NEADL) ${ }^{17}$,

137 which is a widely used self-reported measure of functional mobility with scores ranging

138 from $0-22$, higher scores indicating better ability.

139 Psychological and general health

140 The Stroke and Aphasia Quality of Life Scale (SAQOL-39) ${ }^{18}$ : This is a self-reported

141 measure of quality of life with scores ranging from $0-5$, higher scores indicating better

142 quality of life. The General Health Questionnaire (GHQ-28) ${ }^{19}$ : A self-reported measure

143 used to assess emotional distress in adults. Scores range from $0-28$, with scores above 4

144 indicating the presence of distress. The Pittsburgh Sleep Quality Index (PSQI) ${ }^{20}$ : A self-

145 reported measure with scores ranging from $0-21$, scores of five or greater suggesting

146 poor sleep quality. The Fatigue Assessment Scale (FAS) ${ }^{21}$ : A self-reported measure

147 used to assess chronic fatigue and its interference on mental functioning and activities

148 of daily living. Scores greater or equal to 22 indicating interference from fatigue. ${ }^{22}$

149 Semi-structured evaluation questionnaires and interviews

150 An evaluation questionnaire gathering participants' opinions on the whole experience

151 from recruitment (e.g. how they found out about the programme) to individual sessions 
152 (e.g. were they able to adhere to the exercise protocol) was completed by all

153 participants. These were followed by in-depth semi-structured interviews with

154 participants who were sampled consecutively during the final 2 months of recruitment

$155(\mathrm{n}=15)$. The interview questions were developed by the research team to cover all

156 aspects of the study; they were open ended to promote discussion (see supplementary

157 files for a sample of the interview questions) and were checked for understanding

158 during a pilot with two participants. Similarly, five fitness coaches (one from each

159 participating centre) were interviewed to gather opinions on feasibility and acceptability

160 (e.g. level of support offered to participants during exercise sessions) from the leisure

161 centre's perspective (see supplementary files for a sample of the interview questions).

162 All interviews were conducted by a researcher not directly involved in delivery of the

163 intervention within 3 months of the final exercise programme being completed.

\section{Intervention}

165 The programme was based on the Scottish Government funded 'Best Practice Guidance

166 for the Development of Exercise after Stroke Services in Community Settings ${ }^{23}$. These

167 guidelines advocate the principle of incremental overload adapted to individual needs

168 and goals and broadly follow the FITT (frequency, intensity, type and time) principles,

169 which have been shown to be feasible and effective in stroke populations ${ }^{7}$. The specific

170 content of the eight week programme was determined by a fitness coach following four

171 principles. 1) Frequency: 2-3 sessions per week, 2) Intensity: A moderate level was

172 targeted based on a rating of perceived exertion 24 during the initial fitness test (see

173 below), 3) Type: Cycling was the main feature but other activities could be included for

174 motivational purposes. 4) Time: Increasing duration to a maximum of 30 minutes of

175 cycling per session. 
177 held at each of the five participating centres and the equipment available for participants was standardised across the centres.

\section{Procedure}

181 Following consent participants met with a researcher at home who assisted them in 182 completing the MoCA, SAQOL-39, GHQ-28, PSQI and FAS. Participants were then given an appointment at their nearest participating leisure centre where a fitness coach administered the NEADL and supervised a fitness test comprising cycling with an initial resistance of 25 watts which was increased by 10 watts every 2 minutes until the

186 participant indicated they could not continue or had reached $80 \%$ of their maximal heart

187 rate (208 - $0.7 \mathrm{x}$ age). Based on this, and information regarding stroke related impairments and other conditions, the coach constructed a weekly programme. Participants with insufficient strength to turn one or both pedals were instructed in the use of a MOTOmed ${ }^{\circledR}($ RECK-Technik, Betzenweiler, Germany) assisted bicycle and

191 given progressive targets based on assistance from the bike's motor. The eight week personal training plan was then explained to participants and weekly goals set collaboratively. Participants were provided with an educational pack describing the benefits and side effects of physical activity, as well as practical information about the leisure centre including public transport. They were then left to

196 follow the training plan with supervision from the fitness coaches who had specific

197 qualifications delivering exercise to mobility impaired individuals. Attendance at the

198 leisure centre was recorded using magnetic swipe cards and participants and fitness

199 coaches were given activity logs with weekly individual targets for participants to

200 record their activity. Fitness coaches were asked to record adverse events. After eight 
weeks participants were asked to repeat the outcome measures and an evaluation questionnaire, face-to-face, over the telephone, or by post.

203

204

\section{Analysis procedure}

206 The semi-structured interviews were transcribed and coded using NVivo11 software

207 and analysed using the principles of thematic coding until data saturation was reached ${ }^{25}$.

208 Descriptive statistics and t-tests were used to analyse change in the outcome measures.

209 Results

\section{$210 \quad$ Recruitment and adherence}

211 Recruitment lasted 10 months with a limit of two new participants per week due to the

212 research team's capacity. 115 participants expressed an interest in the study, 38 were 213 not consented as they; decided not to take part (16), did not fulfil the eligibility criteria

214 (15), required better set up of services, including a closer centre or transport difficulties,

215 (5) and expressed an interest after recruitment had ended (2). A total of 77 participants

216 were therefore consented during the recruitment period giving a recruitment rate of 7.7

217 per month. They had a mean age of 63.7 years (SD 12.6, range 21-85) and were 34.4

218 months (SD 46.3, range 5-231), on average, post stroke. 46/77 (60\%) were male, 39/77

219 (51\%) had experienced an Ischaemic stroke, 13/77 (17\%) had a Haemorrhagic stroke 220 and 25/77 (32\%) were unknown. The majority (49/77, 64\%) used some form of walking 221 aid and 9/77 (12\%) were wheelchair users.

From 77 participants who started the programme 66 completed $(86 \%$ retention rate). 11 participants did not complete; 5 failed to attend any session, 3 had ill-health, 2

224 decided they no longer wished to participate and 1 participant died.

\section{Suitability of assessments}


226 Completion rates, at baseline and outcome, were high for the STS (91\%), while only

$22755 \%$ completed the 6MWT. All participants completed the bicycle fitness test with $69 \%$

228 using a recumbent or upright bike and 31\% using the power assisted bicycle.

229 From the evaluation questionnaire, 79\% reported they were able to complete the tests

230 relatively easily, however $6 \%$ reported some difficulties. The interview data produced

231 similar findings with most reporting that they had no issues with the physical

232 assessments. The coaches who were also interviewed were happy with the testing

233 procedures but noted difficulties for the more severely impaired participants to complete

234 the treadmill test.

Completion rates, at baseline and outcome, for the psychological questionnaires

236 ranged from $89 \%$ to $94 \%$. The researcher was present and assisted in the majority of

237 cases, however, a minority chose to complete them on their own with 5\% of participants

238 returning questionnaires with missing items and 3\% not returning them at all. Of the 64

239 participants who completed the eight week evaluation questionnaire, $48 \%$ specifically

240 mentioned that they required help and/or appreciated assistance from the researcher to

241 complete the measures. $40 \%$ stated that they had no issues in particular with the

242 questionnaires but $11 \%$ indicated they had some issue with the more sensitive items in

243 the General Health Questionnaire.

244 Acceptability

245 The programme was acceptable to both ambulatory and non-ambulatory participants.

$24612 \%$ of participants were wheelchair users and $64 \%$ individuals required a mobility aid

247 such as a cane or walking frame.

Almost all (98\%) of participants who completed the evaluation questionnaire

249 responded positively to the programme, stating that it met their needs, and they would

250 recommend it to other stroke survivors. Some potential improvements were given such 
as adapting the programme to include upper limb exercises $(9 \%)$, having more local programmes (5\%) and greater staff awareness of the wider consequences of stroke and related co-morbidities $(5 \%)$.

The vast majority (98\%) of participants felt comfortable in the leisure centre, reporting that there was not much else the staff could have done to improve their experience. Some negative aspects were mentioned, for example, $23 \%$ reported they would have liked improvements in equipment or facilities, $5 \%$ would have like more contact with staff and 3\% reported that better organisation was required.

260 programme was beneficial and support was available from both the research team and

261 fitness coaches:

"Very good, I felt that it was something that I needed and it came just at the right time and it just has made me go from strength to strength so I can't praise it enough" (P111)

"Yes, I did find it helpful, because I think that if I walked into the gym, I would

265 probably would have walked out again" (P67)

266 Adherence

267 Different objectives for the exercise programme were created for participants (see Table

2681 for details). $29 \%(19 / 66)$ of participants aimed to increase duration with $84 \%$ managing to adhere to this, however, there was considerable variation of actual change

270 in cycling time, ranging from 5 to 90 minutes. 29\% (19/66) of participants were given a

271 plan to maintain the same exercise routine for the whole 8 weeks as incrementing was

272 not considered appropriate. None of the plans aimed to increase frequency or the combination of frequency, duration and intensity. 
participants adhered to their plan, however, when asked in the evaluation questionnaire

$27781 \%$ participants reported they had adhered to the programme, and $69 \%$ reported that

278 they enjoyed the goal-oriented aspect of the programme. $22 \%$ reported negative or partially negative responses, with $8 \%$ reporting pain flares up or other physical issues.

The interview data revealed that participants felt the plan had contributed to

281 their adherence with improved motivation through monitoring of progress.

282 "I think I need that discipline. And again I think with a gym, if you've got a tailored

283 plan then that that's your routine - that's what you stick to" (P73)

284 The coaches reported no problems in developing tailored plans. Alterations to the plans

285 were made depending on how the participants felt and this was carried fluidly between

286 coach and participant.

\section{Improvements in fitness and quality of life}

49/77 (64\%) of participants were able to complete the STS test (Table 2) at baseline, $91 \%$ of whom took longer than $12 \mathrm{~s}$, indicating substantial impairment ${ }^{14}$. These scores significantly improved after the programme $($ Mpre $=25.2 \mathrm{~s}$, Mpost $=19.0 \mathrm{~s}, \mathrm{t}(48)=3.28$, $p=.002$ ), with an average reduction in time of $4.81 \mathrm{~s}$. By outcome $18 \%$ of participants

292 had reduced their score to below 12s. Walking speed at baseline was slow (PWS: Mpre $=0.69 \mathrm{~m} / \mathrm{s}$, range $=0.2 \mathrm{~m} / \mathrm{s}-1.9 \mathrm{~m} / \mathrm{s}$ ) and this improved, though not statistically significantly, following the programme $(\mathrm{t}(45)=1.94, \mathrm{p}=.059)$. The distance walked in six minutes (6MWT) was completed by 19 participants and showed no significant change after the programme $(\mathrm{t}(18)=.05, \mathrm{p}=.96)$. Activities of daily living (NEADL), however, did significantly improve (Mpre=12.2, Mpost=13.2, $\mathrm{t}(52)=3.26, \mathrm{p}=.002)$.

The scores for the SAQOL-39 were relatively high at baseline and improved significantly post-programme, with improvements in the subscales of psychosocial functioning $($ Mpre=3.82, Mpost=4.15, $\mathrm{t}(56)=3.42, \mathrm{p}=.001)$ and energy $($ Mpre=3.75, 
301 Mpost $=4.02, \mathrm{t}(57)=2.44, \mathrm{p}=.018)$. Similarly, overall levels of distress were low at

302 baseline and improved significantly $($ Mpre $=6.18$, Mpost $=4.19, \mathrm{t}(56)=2.19, \mathrm{p}=.033)$

303 with significant changes on the depression subscale (Mpre $=.97$, Mpost $=.55, \mathrm{t}(57)=2.70$,

$304 \mathrm{p}=.009$ ) but not the other subscales. Reasonably good baseline sleep scores (PSQI) did

305 not suggest extreme sleep problems, however, $63 \%$ of participants had a score of 5 or

306 more at baseline indicating poor sleep quality. Overall significant changes in PSQI

307 scores were not found, but further analyses of ambulatory participants showed a

308 significant improvement $(\mathrm{t}(50)=2.27, \mathrm{p}=.028)$ for this sub-group.

309 Finally, the FAS showed $31 \%$ of participants were experiencing substantial or

310 extreme fatigue at baseline and there was no overall significant improvements in this

311 following the programme. Some participants did report changes though:

312 "What I am finding more is that I'm sleeping more at night, basically when I go to bed I

313 usually hit the pillow and I'm out anyway, but I think it's mainly because of the

314 exercise.” (P29)

315 Seven adverse events were noted over the recruitment period, two attributable to

316 the study involving minor injury through falling off a treadmill.

\section{Discussion}

318 This quasi-experimental study was conducted primarily to test the feasibility and

319 acceptability of a physical activity programme that was designed to be sustainable

320 through the use of existing local government facilities and accessible to all stroke

321 survivors by using standard and powered cycling ergometers.

322 The programme was popular, with 115 individuals expressing an interest,

323 representing around $1 \%$ of the estimated stroke population living within the NHS

324 Lanarkshire health board area. A promising outcome from the recruitment process was

325 the number of non-ambulatory participants $(n=9)$, which contrasts with most other 
studies of physical activity in stroke populations which typically include individuals

327 with at least minimal ambulatory ability ${ }^{26}$. In this regard the study is in line with guidelines for wider access to physical activity for stroke survivors ${ }^{27}$.

The recruitment figures support the use of leisure centres for delivering physical

330 activity classes to the whole stroke survivor population, and the leisure centre staff also

331 found the programme to be feasible and acceptable. It should be noted however that the

332 average age of participants (63.37 years) was relatively young, suggesting the

333 programme may be less appealing to older people or that the stroke liaison nurse was

334 less likely to approach them.

The high level of adherence ( $81 \%)$ to the programme was a very positive outcome. While exercise adherence is not well reported in stroke studies ${ }^{8}$ it is generally considered to be low, for example Miller et al. ${ }^{28}$ report a $65 \%$ adherence rate.

This reinforces the benefits of using local leisure centres which not only have fitness coaches to supervise the activities but a range of exercise equipment to tailor the

340 programme to the changing needs of the individual. The social reward in attending the

341 centre may also have contributed to this high adherence rate.

342 In terms of the assessment measures these were acceptable except for the high 343 numbers who could not complete the treadmill six minute walk test. It should be noted

344 that the completion of questionnaires typically required assistance and some participants

345 raised concerns about the more sensitive elements of the General Health Questionnaire.

346 The statistically significant improvements in STS ability are consistent with the

347 principle of training specificity as the motor pattern of recumbent cycling and STS are

348 similar ${ }^{11}$. Upright cycling has a motor pattern considered comparable to walking ${ }^{29}$ and,

349 if used, might have promoted greater recovery of walking function. While these 
350 improvements did not impact on walking function, the improved STS capacity is

351 nevertheless a valuable outcome given the importance of this movement in daily life ${ }^{30}$.

352 Significant improvements to quality of life and general health, specifically

353 psychosocial functioning, energy and depression, were encouraging as these can be

354 difficult to alter following stroke $\mathrm{e}^{31,32}$. The improvement in sleep quality in the

355 ambulatory participants was also positive as other reports have indicated that up to $37 \%$

356 of stroke survivors experience insomnia one year post-stroke ${ }^{33}$. However, it should be

357 noted that the study participants did not demonstrate particularly poor levels of quality

358 of life, distress or sleep at baseline.

359 Study limitations

360 The study had a number of limitations; firstly the activity logs and entrance swipe card

361 monitoring used in this study did not yield sufficiently reliable data, more efficacious

362 ways of tracking exercise participation should be considered. Secondly, similar to

363 previous studies, this study attracted younger participants with relatively high

364 psychosocial functioning compromising external validity to the wider stroke

365 community. Thirdly, using treadmills was too demanding for many participants and

366 were related to the two related adverse events.

\section{Conclusions}

368 The most important findings of this study were the willingness of participants to

369 undertake an exercise programme in their local leisure centre despite ongoing disability.

370 The study achieved its primary goal of testing the acceptability and feasibility of this

371 method of exercise delivery in the local community using recumbent cycle ergometers

372 as the focus of the programme due to their close similarity in the motor pattern of the

373 STS movement and their general accessibility. 
377 Data availability statement

378 The data that support the findings of this study are available on request from the

379 corresponding author, $[\mathrm{AK}]$. The data are not publicly available due to restrictions on

380 information that could compromise the privacy of research participants.

381

382 Acknowledgements

383 The study was funded by the Chief Scientist's Office and supported by North and South

384 Lanarkshire Leisure services.

385

386 References

387

388 1. Quaney BM, Boyd LA, McDowd JM, Zahner LH, Jianghua H, Mayo MS,

389 Macko RF. Aerobic Exercise Improves Cognition and Motor Function Poststroke.

390 Neurorehabilitation and Neural Repair. 2009;23(9):879-885.

391 2. Cumming T, Tyedin K, Churilov L, Morris M, Bernhardt J. The effect of

392 physical activity on cognitive function after stroke: A systematic review. International

393 Psychogeriatrics. 2012;24(4):557-567.

394 3. Chen MD, Rimmer JH. Effects of exercise on uality of life in stroke survivors:

395 A meta-analysis. Stroke. 2011;42(3):832-837.

396 4. Pang MYC, Eng JJ, Dawson AS, Gylfadóttir S. The use of aerobic exercise

397 training in improving aerobic capacity in individuals with stroke: a meta-analysis.

398 Clinical Rehabilitation. 2006;20(2):97-111. 
399 5. Bernhardt J, Dewey H, Thrift A, Donnan G. Inactive and alone - Physical

400 activity within the first 14 days of acute stroke unit care. Stroke. 2004;35(4):1005-1009.

401 6. Kerr A, Rowe P, Esson D, Barber M. Changes in the physical activity of acute

402 stroke survivors between inpatient and community living with early supported

403 discharge: an observational cohort study. Physiotherapy. 2016;102(4):327-331.

404 7. Mead GE, Greig CA, Cunningham I, Lewis SJ, Dinan S, Saunders DH,

405 Fitzsimons C, Young A. Stroke: A randomized trial of exercise or relaxation. Journal Of

406 The American Geriatrics Society. 2007;55(6):892-899.

407 8. Jurkiewicz MT, Marzolini S, Oh P. Adherence to a Home-Based Exercise

408 Program for Individuals After Stroke. Topics in Stroke Rehabilitation. 2011;18(3):277-

409284.

410 9. Tang A, Sibley KM, Thomas SG, Bayley MT, Richardson D, McIlroy WE,

411 Brooks D. Effects of an aerobic exercise program on aerobic capacity, spatiotemporal

412 gait parameters, and functional capacity in subacute stroke. Neurorehabilitation and

413 neural repair. 2009;23(4):398-406.

414 10. Hancock NJ, Shepstone L, Winterbotham W, Pomeroy V. Effects of lower limb

415 reciprocal pedalling exercise on motor function after stroke: a systematic review of

416 randomized and nonrandomized studies. International Journal of Stroke. 2012;7(1):47-

41760.

418 11. Kerr A, Rafferty D, Moffat F, Morlan G. Specificity of recumbent cycling as a

419 training modality for the functional movements; sit-to-stand and step-up. Clinical

420 Biomechanics. 2007;22 (10):1104-1111.

421 12. MRC. https://mrc.ukri.org/documents/pdf/developing-and-evaluating-complex422 interventions/. 2006. 
423 13. Nasreddine ZS, Phillips NA, Bedirian V, Charbonneau S, Whitehead V, Collin I,

424 Cummings JL, Chertkow H. The montreal cognitive assessment, MoCA: A brief 425 screening tool for mild cognitive impairment. Journal of the American Geriatrics 426 Society. 2005;53(4):695-699.

427 14. Mong Y, Teo TW, Ng SS. 5-Repetition Sit-to-Stand Test in Subjects With 428 Chronic Stroke: Reliability and Validity. Archives of Physical Medicine and 429 Rehabilitation. 2010;91(3):407-413.

430 15. Kao P-C, Dingwell JB, Higginson JS, Binder-Macleod S. Dynamic instability 431 during post-stroke hemiparetic walking. Gait \& posture. 2014;40(3):457-463.

432 16. Laskin JJ, Bundy S, Marron H, Moore H, Swanson M, Blair M, Humphrey R. 433 Using a treadmill for the 6-minute walk test: reliability and validity. Journal of 434 cardiopulmonary rehabilitation and prevention. 2007;27(6):407-410.

435 17. Nouri FM, Lincoln NB. An extended activities of daily living scale for stroke 436 patients. Clinical Rehabilitation. 1987;1(4):301-305.

437 18. Hilari K, Byng S, Lamping DL, Smith SC. Stroke and aphasia quality of life 438 scale-39 (SAQOL-39) - Evaluation of acceptability, reliability, and validity. Stroke. $4392003 ; 34(8): 1944-1950$.

440 19. Goldberg DP, Hillier VF. Scaled version of the General Health Questionnaire. 441 Psychological Medicine. 1979;9(1):139-145.

442 20. Buysse DJ, Reynolds CF, Monk TH, Berman SR, Kupfer DJ. The Pittsburgh 443 Sleep Quality Index - a new instrument for psychiatric practice and research. Psychiatry 444 Research. 1989;28(2):193-213.

445 21. Mead G, Lynch J, Greig C, Young A, Lewis S, Sharpe M. Evaluation of fatigue 446 scales in stroke patients. Stroke. 2007;38(7):2090-2095. 
22. Michielsen HJ, De Vries J, Van Heck GL. Psychometric qualities of a brief self-

448 rated fatigue measure The Fatigue Assessment Scale. Journal of Psychosomatic

449 Research. 2003;54(4):345-352.

450 23. Best C, van Wijck F, Dinan-Young S, Dennis J, Smith M, Fraser H, Donaghy

451 M, Mead G. Best Practice Guidance for the Development of Exercise after Stroke

452 Services in Community Settings.

453 http://www.exerciseafterstroke.org.uk/resources/Exercise_After_Stroke_Guidelines.pdf.

454 24. Borg GAV. Psychophysical bases of perceived exertion. Medicine and Science

455 in Sport and Exercise. 1982;14(5):377-381.

456 25. Braun V, Clark V. Using thematic analysis in psychology. Qualitative Research

457 in Psychology. 2006;3:77-101.

458 26. Stoller O, de Bruin ED, Knols RH, Hunt KJ. Effects of cardiovascular exercise 459 early after stroke: systematic review and meta-analysis. BMC Neurology. 2012;12:4546045.

461 27. Mead G. Exercise After Stroke. British Medical Journal. 2009; 339: 2795

462 28. Miller, K.K., Porter, R.E., DeBaun-Sprague, E., Van Puymbroeck, M. and 463 Schmid, A.A. Exercise after stroke: patient adherence and beliefs after discharge from 464 rehabilitation. Topics in Stroke Rehabilitation. 2017 24(2), pp.142-148.

465 29. Hancock NJ, Shepstone L, Rowe P, Myint PK, Pomeroy VM. Towards Upright 466 Pedalling to drive recovery in people who cannot walk in the first weeks after stroke:

467 movement patterns and measurement. Physiotherapy. 2017;103(4):400-406.

468 30. Dall PM, Kerr A. Frequency of the sit to stand task: An observational study of 469 free-living adults. Applied Ergonomics. 2010;41(1):58-61.

470 31. Haley WE, Roth DL, Kissela B, Perkins M, Howard G. Quality of life after 471 stroke: a prospective longitudinal study. Quality of Life Research. 2011;20(6):799-806. 
472 32. Choi-Kwon S, Kim JS. Poststroke Fatigue: An Emerging, Critical Issue in

473 Stroke Medicine. International Journal of Stroke. 2011;6(4):328-336.

474 33. Glozier N, Moullaali TJ, Sivertsen B, Kim D, Mead G, Jan S, Li Q, Hackett ML.

475 The Course and Impact of Poststroke Insomnia in Stroke Survivors Aged 18 to 65

476 Years: Results from the Psychosocial Outcomes In StrokE (POISE) Study.

477 Cerebrovascular Diseases Extra. 2017;7(1):9-20.

478

479 
480 Table 1: Programme objectives and percentage of participants $(n=66)$ who followed

481 them

482

\begin{tabular}{lccc}
\hline & Objective & Followed plan & \% Who followed \\
\hline Duration & $\uparrow 19$ & 16 & 84 \\
Intensity & $\uparrow 2$ & 1 & 50 \\
Frequency & $\uparrow 0$ & & 64 \\
Duration and intensity & $\uparrow 11$ & 7 & 100 \\
Duration and frequency & $\uparrow 0$ & & 68 \\
Intensity and frequency & $\uparrow 1$ & 1 & \\
Duration, intensity and frequency & $\uparrow 0$ & & 58 \\
Maintain & $\uparrow 19$ & 13 & \\
Programme not returned & 14 & Not known & \\
Total & 66 & 38 & \\
\hline
\end{tabular}

483

484 Table 2: Outcome measures at baseline and following programme (outcome)

\begin{tabular}{llll}
\hline Test & Baseline & Outcome & $\mathrm{p}$ \\
& Mean (SD) & Mean (SD) & \\
\hline STS (s), $\mathrm{n}=49$ & $25.2(20.2)$ & $19.0(10.9)$ & .002 \\
Preferred walking speed (m/s), $\mathrm{n}=46$ & $0.7(0.4)$ & $0.9(0.5)$ & .059 \\
6 minute walk test (m), $\mathrm{n}=20$ & $327.7(228.9)$ & $325.5(292.2)$ & .960 \\
NEADL, n=53 & $12.2(6.1)$ & $13.2(6.4)$ & .002 \\
SAQOL-39 Total, $\mathrm{n}=55$ & $3.9(0.7)$ & $4.1(0.7)$ & .007 \\
GHQ-28 Total, $\mathrm{n}=57$ & $6.2(4.7)$ & $5.0(3.9)$ & .033 \\
PSQI, $\mathrm{n}=56$ & $6.2(4.4)$ & $5.3(3.8)$ & .071 \\
Fatigue Assessment Scale, $\mathrm{n}=57$ & $19.9(8.8)$ & $18.8(7.3)$ & .120
\end{tabular}

